\title{
HIV testing for the non-specialist
}

\author{
M Hamill ${ }^{1,2}$ S Wright ${ }^{2,3} \quad$ K Kemp $^{4} \quad$ S de Silva ${ }^{2,5}$
}

J R Soc Med 2006;99:128-131

\section{INTRODUCTION}

Since the introduction of highly active antiretroviral therapy the landscape of HIV has changed dramatically with greatly improved prognosis. ${ }^{1}$ The incidence of HIV infections continues to rise, ${ }^{2}$ with many patients undiagnosed until they present with a potentially preventable late complication or opportunistic infection. Current Health Protection Agency estimates are of 58300 cases of HIV in the UK, a third of whom have not yet been diagnosed. Of these, $71 \%$ of infections are in adults aged 15-39 years. ${ }^{3}$ Many of those at risk of HIV will present to a variety of services - in particular accident and emergency, psychiatry, primary care, drug and alcohol services, genito-urinary medicine and other medical specialities. There are already examples of services where HIV testing is seen as a 'routine' test (e.g. in obstetrics and genito-urinary medicine); this needs to be emulated and built upon (Box 1). The key to earlier diagnosis and treatment is to increase HIV testing uptake by the demystification of testing and a high clinical index of suspicion. This will involve consideration of testing of those not in the traditional high-risk groups.

If HIV disease is not considered early then it may be overlooked until the patient presents with advanced disease or with an opportunistic infection. Late presenters, following multiple presentations to medical services, remain a common problem. ${ }^{4}$

\section{HISTORY}

History taking, in terms of risk factors for HIV, is central to correctly identifying those who warrant a test. This will also help to decrease anxiety in those at low risk. In order to establish increased risk of HIV infection it is important to establish if the classical high-risk behaviours are present. Individuals, or their sexual contacts, from an area with high HIV prevalence (Sub Saharan Africa, Caribbean, Eastern Europe, South Asia and South America) remain numerically the greatest risk. ${ }^{3}$ Sub Saharan Africa is a well known area of high prevalence: however, the Caribbean and India have

${ }^{1}$ Institute of Social and Cultural Anthropology, University of Oxford, Oxford OX2 6PE; ${ }^{2}$ The Mortimer Market Centre, Camden PCT, London WC1E 6AU; ${ }^{3}$ Deputy Head of Health Advising Services, Archway Sexual Health Centre, Camden PCT, London N19 5NF;

${ }^{4}$ Lead Clinician in Primary Health Care, The Primary Care Unit, London NW1 2LS;

\section{Box 1 Reasons for offering HIV tests}

- Increasing incidence of HIV infection

- Greatly improved prognosis with timely treatment

- Detection of asymptomatic HIV disease, therefore avoiding AIDS diagnoses

- Poor perception of risk of infection by patients and healthcare professionals

- Offering a HIV test increases awareness and may alter behaviour if health promotion advice is given

- Preventing onward transmission and mother to child transmission

- Increasing participation of patients in their treatment goals.

less well-publicized epidemics. Current or ex-intravenous drug users are at increased risk of all blood borne viral infections including HIV. A history of commercial sex work (prostitution) or blood transfusion/operation in a developing country also needs to be explored. Many will not readily volunteer a history of intravenous drug use or commercial sex work: this, therefore, needs to be specifically questioned in order to maximize opportunities for health promotion as well as testing and vaccination. Traditionally, in the UK, the highest risk group were gay or bisexual men. They still represent the highest number of diagnoses infected within the UK: however, the absolute annual incidence is now highest in heterosexuals, representing imported infection from endemic areas. ${ }^{3}$

The history may establish if symptoms of weight loss, fever, cough or other localizing symptoms that may suggest immunosuppression are present. Symptoms suggestive of other common illness for example diabetes or malignancy may also need to be assessed; of course multiple pathologies may coexist particularly in an ageing population (Box 2). ${ }^{5}$

\section{EXAMINATION}

When considering a diagnosis of HIV it is important to look for signs of immunosuppression. This should include documenting weight loss and careful examination of the oral cavity for candida, oral hairy leukoplakia or palatal Kaposi's sarcoma. Skin should be inspected for evidence of shingles, severe seborrhaeic dermatitis, marked recurrent herpetic ulcers and Kaposi's sarcoma. Lymph node examination may reveal generalised lymphadenopathy or isolated enlarged regional nodes. Finally hepatospenomegaly or retinitis on fundoscopy may be the only sign of 


\section{Box 2 Hypothetical case example}

\begin{abstract}
A 50-year-old woman is referred to medical outpatients by her GP. She reports weight loss and lymphadenopathy. She had a 'terrible flu' 12 months earlier; a full blood count taken at that time revealed low platelets and low total lymphocyte count which resolved spontaneously. She is worried about malignancy as her ex-husband died from 'cancer' 6 months previously. This was in the Caribbean so you have no details of his diagnosis. She has always been well other than mild hypertension. Currently she has no localizing symptoms to suggest a diagnosis.
\end{abstract}

\section{Issues raised by this case}

In order to correctly diagnose HIV infection it is essential to have a high index of suspicion. HIV affects all ages, from infants to the elderly, races and social classes and may present many years after sexual activity has ceased. Unless an appropriate history is taken then subtle clues may be missed. In particular, risk factors for HIV infection need to be directly addressed for example sexual history, injecting drug use, etc. It raises the important consideration that HIV is increasingly prevalent outside the traditional risk groups of gay men and sub Saharan Africans. Delay in diagnosing HIV may have adverse effects on the patient's health.

Many of the HIV tests that you organize may be negative; however this still may be a very useful investigation.

immunosuppression and may suggest the presence of an opportunistic infection.

\section{INVESTIGATIONS}

Either prior to, or simultaneously with, considering an HIV diagnosis, it is important to exclude common pathology by simple investigations; e.g. serum glucose, urea and electrolytes, liver and thyroid function tests, calcium, full blood count, faecal occult bloods, chest X-ray, etc. All preliminary tests may be negative or inconclusive. Be alerted to the possibility of HIV infection by the presence of lymphopaenia, thrombocytopenia or a normochromic, normocytic anaemia on full blood count. It is possible that HIV may coexist with other chronic conditions and needs to be considered and screened for if there is a clinical concern. $^{5}$

\section{OFFERING THE HIV TEST}

Although the benefits of early diagnosis are apparent, many healthcare professionals feel ill equipped to raise the issue of HIV testing. 6 The following structure may increase confidence in offering testing.

Obtain consent, ensure the patient understands that you plan to request this test, and that they understand what it involves (Box 3). ${ }^{7}$ Confidentiality must be stressed, as many patients will have much anxiety around this issue. In the past, insurance and mortgage companies have asked questions relating to life style to try to identify people more likely to have adverse health events. In cases with perceived increased risk for HIV there may have been increased premiums or refusal to offer life insurance. Since 2004, the insurance ombudsman has directed all insurance companies in the UK to no longer routinely ask any questions related to lifestyle (such as sexual orientation) and such questions are now only permitted if the applicant has ever tested positive for HIV. Nor are insurers allowed to take into consideration previous HIV tests if the results of these were negative. Patients may obtain free, anonymous HIV testing from genitourinary medicine clinics although most such services will strongly encourage correspondence with the general practitioner/referring clinician if the test proves positive. If HIV testing is undertaken by the GP, he/ she may be obliged to disclose the results to insurers if the patient has agreed that the insurance or mortgage company may approach the GP for a medical report. In order to demystify HIV testing; explain that HIV testing is a 'standard' test designed to detect a treatable (albeit incurable) infectious disease that, if successfully managed, can have an excellent prognosis. This can be facilitated by an explanation that all pregnant women are offered HIV testing routinely during pregnancy.

It is important to explain that, in some cases, HIV may be a cause of the patient's symptoms and that it therefore needs to be excluded, or diagnosed and treated. Analogies with other chronic diseases for example diabetes may be helpful in enabling patients to see HIV in context. The prevalence of HIV in certain population groups such as those from endemic areas as well as in gay/bisexual men, commercial sex workers, intravenous drug users and the recipients of blood transfusions outside the developed world should also be discussed. This enables patients and those testing them to understand the need for testing and may help to reduce the stigma of undertaking a HIV test. Discuss the likelihood, or relative risk, of a positive test (as far as can be ascertained) and discuss what support and services may be available should the test be positive. For particular groups, such as Africans, HIV remains hugely stigmatizing, therefore providing information on dedicated support groups such as Positively Women may help improve uptake of testing. ${ }^{8}$

Box 3 Key points to remember when offering HIV testing

- Consent

- Confidentiality

- Explain the reasons behind the test

- Discuss the probability of a positive result

- Establish risk factors for HIV acquisition

- Emphasize the benefits of early diagnosis and ongoing health promotion. 
Testing may not be reliable if HIV infection has been acquired relatively recently as the test relies on antibody production; but most patients will have a positive test result if infected more than 3 months before. Emphasize that in the UK, treatment for HIV is now relatively simple for those who test positive, but will involve regular blood tests and may require them to take medication on a daily basis. This will depend on the stage of HIV infection and degree of immune suppression. It is also helpful to explain that effective treatment will reduce the risks of transmission to others - pregnant women who are HIV positive may deliver babies free of infection if their disease is correctly managed. ${ }^{9,10}$

Remember that to discuss HIV testing you will use communication skills that healthcare professionals utilize on a daily basis when discussing screening for other serious diseases. These skills are particularly pertinent if a rapid HIV test is being employed.

Any appropriately trained healthcare worker can offer HIV testing as long as they are able to discuss the above issues. It is crucial to ascertain the patients' understanding of the test and his/her expectation of the result. As with other areas of medicine, good documentation is essential: however, this process need not be lengthy or time consuming. All staff must be non-judgemental especially when dealing with sensitive personal information such as sexuality or involvement in commercial sex work.

\section{WHAT TO DO: PRACTICAL CONSIDERATIONS}

Send a serology sample, i.e. clotted blood (or plasma) for HIV antibody testing (the same sample can also be used to test for syphilis and hepatitis B and C if appropriate). Some laboratories prefer a plasma sample, therefore liaise with your local laboratory, they will also arrange to process the test urgently if requested. Urgent results can usually be available within 24 hours, to facilitate this ensure that you have the name and contact number of your local virology consultant.

As some patients will not return for their results ensure that you have an up-to-date mobile telephone number and address for all patients upon whom testing is undertaken. ${ }^{11}$

In order that you feel prepared for the follow-up visit, make sure that you have the result of the test before the patient's follow-up appointment. Aim to give the patient an appointment with the same person who offered the test; ideally give a double slot if the test is likely to be positive and suggest that they bring somebody along with them. In order to decrease anxiety give the result straight away: do not increase anxiety by avoidance and preamble. Always attempt to have a hard copy of the result available, it helps patients to absorb the information and allows clarification
Ensure that you have a private, quiet room to give the result. Switch off your mobile telephone or bleep and ask not to be disturbed during the consultation. It is often helpful to have a member of the nursing staff present. Make sure you have booked a translator if the patient does not speak English well in order that you can fully explain the result and its implications.

Allow the patient silence and time to absorb the information: it is common that patients given a positive result are emotionally shocked. Although hysterical reactions are anticipated and feared by medical staff they are uncommon and can generally be managed in a calm, supportive environment.

If the patient tests HIV positive arrange to refer them to local HIV/genitourinary medicine services. Have ready the name and details of the HIV consultant or nurse specialist who will arrange an appointment, usually within 2 weeks. ${ }^{12}$ At this visit parameters such as CD4 count and viral load will be established; it is not necessary to undertake these tests prior to this. Providing an explanation of these initial, practical considerations help patients to assume some control over their condition. If they are particularly distressed, a health advisor from the local genitourinary medicine/HIV service can arrange to see them urgently for psychological and practical support (e.g. the testing of their partner or children). Offer to see the patient again in the interim if appropriate.

Do not feel that you have to answer all questions at this stage but do reiterate that HIV is not synonymous with AIDS, is treatable and compatible with a good quality of life. Offer patients written information/leaflets about HIV infection, support and advice services, etc., that they make take away and refer to later if they wish to. Most patients do not expect, or want, an encyclopaedic explanation of HIV at the time of diagnosis.

An alternative consideration is that truly 'routine' testing involves testing without an obligatory risk history, and without regard to sexual orientation, race, ethnicity or gender. Clinicians can simply ask all sexually active patients if they are interested in HIV testing. This allows testing of those not in traditional 'at risk' groups thereby improving detection of HIV. ${ }^{13}$

\section{Other suggestions}

If you wish to acquire more skills in HIV testing then arrange to sit in at a local genitourinary medicine clinic as an observer. There is also a 2-3 day Sexually Transmitted Infection Foundation course (see BASHH website [www.bash.org/]) that is useful for all healthcare professionals. ${ }^{12}$ Display posters in outpatient waiting rooms and in other visible places to raise awareness of HIV testing, these should be displayed in various languages. 


\section{USEFUL WEBSITES}

- British Association for Sexual Health and HIV [www.bashh.org] for a list of genitourinary medicine clinics, guidelines and details of Sexually Transmitted Infection Foundation courses

- The Health Protection Agency [www.hpa.org.uk/ infections/topics_az/hiv_and_sti/hiv/hiv.htm] for latest data on HIV

- Medical foundation for HIV \& Sexual Health [www. medfash.org.uk] for their HIV in Primary Care publication [www.medfash.org.uk/publications/documents / HIV_in_Primary_Care.pdf]

- Terence Higgins Trust [www.tht.org.uk/other_orgs/ other_orgs.htm] for mortgage, financial, life assurance and travel insurance advice for HIV positive individuals

- British HIV Association [www.bhiva.org/] for HIV updates and treatment guidelines

\section{REFERENCES}

1 Palella FJ Jr, Delaney KM, Moorman AC, et al. Declining morbidity and mortality among patients with advanced human immunodeficiency virus infection. HIV outpatients study investigators $N$ Engl J Med 1998; $338: 853-60$
2 AIDS epidemic update 2003, UNAIDS [www.unaids.org/Unaids/ EN/Resources/Publications/corporate+ publications/aids+epidemic + update+-+december+2003.asp] Accessed August 2005

3 Health Protection Agency [www.hpa.org.uk/infections/topics_az/ hiv_and_sti/hiv/hiv.htm] accessed August 2005

4 Burns FM, Fakoya AO, Copas AJ, et al. Africans in London continue to present with advanced HIV disease in the era of highly active antiretroviral therapy AIDS 2001;15:2453-5

5 Inelman EM, Gasparini G, Enzi G. HIV/AIDS in older adults: a case report and literature review. Geriatrics 2005;60:26-30

6 Nichols SA, Bhatta MP, Lewis J, et al. Prenatal HIV counselling, testing, and antiretroviral prophylaxis by obstetric and family medicine providers in Alabama Am J Med Sci 2002;324:305-9

7 [www.bashh.org/guidelines/draft_04/hiv_testing_2005_draft5.doc] Accessed August 2005

8 Positively Women [http://www.positivelywomen.org.uk] Accessed August 2005

9 Brocklehurst P, Volmink J. Antiretrovirals for reducing the risk of mother-to-child transmission of HIV infection. Cochrane Database Syst Rev 2002;2:CD003510

10 Thorne C, Newell ML Mother-to-child transmission of HIV infection and its prevention. Curr HIV Res 2003;1:447-62

11 Downer SR, Meara JG, Da Costa AC. Use of SMS text messaging to improve outpatient attendance. Med J Aust 2005; 183:366-8

12 [http://www.bashh.org/directory.htm] Accessed August 2005

13 Coker R. Compulsory screening of immigrants for tuberculosis and HIV. BMJ 2004;328:298-300 